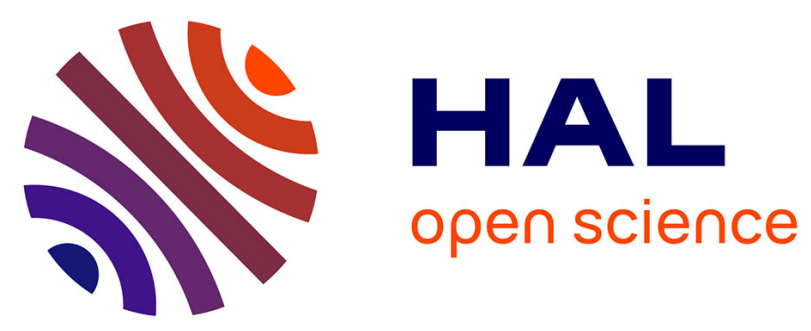

\title{
Assessment of shear-dependent kinetics of primary haemostasis with a microfluidic acoustic biosensor
}

Aleksandr Oseev, Thomas Pierre Lecompte, Fabien Remy-Martin, Guillaume

Mourey, Franck Chollet, Benoit Le Roy de Boiseaumarié, Alain Rouleau, Ophélie Bourgeois, Emmanuel de Maistre, Céline Elie-Caille, et al.

\section{To cite this version:}

Aleksandr Oseev, Thomas Pierre Lecompte, Fabien Remy-Martin, Guillaume Mourey, Franck Chollet, et al.. Assessment of shear-dependent kinetics of primary haemostasis with a microfluidic acoustic biosensor. IEEE Transactions on Biomedical Engineering, 2020, 68 (8), pp.2329-2338. hal-03360746

\author{
HAL Id: hal-03360746 \\ https://hal.science/hal-03360746
}

Submitted on 1 Oct 2021

HAL is a multi-disciplinary open access archive for the deposit and dissemination of scientific research documents, whether they are published or not. The documents may come from teaching and research institutions in France or abroad, or from public or private research centers.
L'archive ouverte pluridisciplinaire $\mathbf{H A L}$, est destinée au dépôt et à la diffusion de documents scientifiques de niveau recherche, publiés ou non, émanant des établissements d'enseignement et de recherche français ou étrangers, des laboratoires publics ou privés. 


\title{
Assessment of shear-dependent kinetics of primary haemostasis with a microfluidic acoustic biosensor
}

\author{
Aleksandr Oseev, Thomas Lecompte, Fabien Remy-Martin, Guillaume Mourey, Franck Chollet, \\ Benoît Le Roy de Boiseaumarié, Alain Rouleau, Ophélie Bourgeois, Emmanuel de Maistre, Céline \\ Élie-Caille, Jean-François Manceau, Wilfrid Boireau, and Thérèse Leblois
}

\begin{abstract}
Goal: Primary haemostasis is a complex dynamic process, which involves in-flow interactions between platelets and sub-endothelial matrix at the area of the damaged vessel wall. It results in a first haemostatic plug, which stops bleeding, before coagulation ensues and consolidates it. The diagnosis of primary haemostasis defect would benefit from evaluation of the whole sequence of mechanisms involved in platelet plug formation in flow. This work proposes a new approach that is based on characterization of the shear-dependent kinetics that enables the evaluation of the early stages of primary haemostasis. We used a label-free method with a quartz crystal microbalance (QCM) biosensor to measure the platelet deposits over time onto covalently immobilized type I fibrillar collagen. We defined three metrics: total frequency shift, lag time, and growth rate. The measurement was completed at four predefined shear rates prevailing in small vessels $\left(500,770,1000\right.$ and $\left.1500 \mathrm{~s}^{-1}\right)$ during five minutes of perfusion with anticoagulated normal whole blood. The rate of the frequency shift over the first five minutes was strongly influenced by shear rate conditions, presenting a maximum around $770 \mathrm{~s}^{-1}$, and varying by a factor larger than three in the studied shear rate range. To validate the biosensor signal, the total frequency shift was compared to results obtained by atomic force microscopy (AFM) on final platelet deposits. The results show that shear-dependent kinetic assays are promising as an advanced method for screening of primary haemostasis.
\end{abstract}

Index Terms-Acoustic biosensor, microfluidics, QCM, primary haemostasis, platelet, shear rate

\section{INTRODUCTION}

$\mathrm{H}$ AEMOSTASIS is the blood response at sites of vessel injury that aims at stopping bleeding, initiated by the contact of blood on sub-endothelial components [1]. Primary haemostasis is the initial step when blood platelets are deposited at the damaged area and form a plug that seals the vascular disruption in a matter of a few minutes. Coagulation ensues to

This research was funded by ANR GHOST $n^{\circ}$ ANR-17-CE19-0026-01, the Interreg V France-Suisse 2014-2020 BLOODE convention $\mathrm{n}^{\circ} 1792$ and the EUR EIPHI ANR-17-EUR-0002. This work was partly supported by the French RENATECH network and its FEMTO-ST technological facility. The fabrication part of the work was assisted by FEMTO-Engineering. Authors would like to thank Rabah Zeggari for his valuable contribution to this work.

*A. Oseev, F. Remy-Martin, F. Chollet, B. Le Roy de Boiseaumarié, A. Rouleau, O. Bourgeois, C. Élie-Caille, J.-F. Manceau, W. Boireau and T. Leblois are with the FEMTO-ST Institute, CNRS UMR-6174, Univ. Bourgogne Franche-Comté, Besançon, France. T. Lecompte is with Geneva University secure the sealing. Platelet plug formation is built up during several consecutive stages: adhesion - platelet interaction with collagen and other matrix components, platelet activation, leading to spreading and further recruitment of more platelets and their aggregation. This dynamic process is markedly influenced by the rheological conditions. In-vitro evaluation of primary haemostasis has to be performed with whole blood (since viscosity, in-flow cells distribution and other aspects of red blood cells play a definite role) at relevant flow conditions with controlled wall shear rates (later referred to as shear rate) in a purposely-designed perfusion chamber. The conditions for blood perfusion should approach the ones occurring in-vivo in small vessels. Assessment over a range of shear rates approximates the various in-vivo conditions, thus potentially enabling a deeper investigation of any possible abnormalities of primary haemostasis [2]. A thorough, accurate and easy-toperform assessment would be an asset, particularly if it could avoid all extra process usually needed for platelet plug observation (like platelet labelling, fixation, washing, etc.). As far as we know, just one device working with flowing blood has been clinically used: PFA (Platelet Function Analyzer, Siemens Healthineers) [3]. It was shown to be sensitive to most of von Willebrand factor dysfunctions but to be limited for the detection of many disturbances in platelet function. Blood is aspirated through a small aperture in a membrane soaked with collagen and a platelet activator, either ADP or epinephrine, and time to occlusion is measured. Highly artificial perfusion chamber design, uncertain and presumably very high shear rate, and poor measurement repeatability are the major drawbacks of PFA [4]. Those drawbacks are to a large extent shared by other flow assays like the T-TAS, which poorly mimic the conditions prevailing in-vivo [5]. There is thus a persisting need for an analytical instrument that approaches the in-vivo conditions in

(faculty of medicine, Geneva Platelet Group), and University Hospital HUG (Haemostasis Unit), Geneva, Switzerland. G. Mourey is affiliated with EFS BFC, Haemostasis Division, Laboratoire de Biologie Médicale et de Greffe, Besançon, France; University Hospital of Besançon, Clinical Haemostasis Unit, Besançon, France; and Université Bourgogne Franche-Comté, INSERM, EFS BFC, UMR1098, Interactions Hôte-Greffon-Tumeur/Ingénierie Cellulaire et Génique, Besançon, France. E. de Maistre is with Centre Hospitalier Universitaire de Dijon-Bourgogne, Haemostasis Lab, Dijon, France.

(correspondence e-mail: aleksandr.oseev@femto-st.fr). 
a closer manner than existing solutions do.

Real-time monitoring of platelet plug formation beyond mere adhesion to subendothelium matrix (and hence coverage) with direct sensing methods is still the Holy Grail. The acoustic biosensor approach is one of the methods that enable direct measurement of biological elements interacting with the biointerface. In this contribution, we utilized an acoustic biosensor approach with Quartz Crystal Microbalance (QCM) operating at fundamental thickness shear vibration mode. The biosensor response was measured as a resonance frequency shift, which depends on the amount of biological (platelet) deposits at the biointerface.

Previous works with QCM based biosensors addressed some aspects of haemostasis: platelet aggregation, which is only one part of the platelet response to achieve haemostasis [6], [7]. A QCM based sensor approach was also used to study the interactions between immobilized $\mathrm{vWF}$ and the recombinant form of its platelet surface receptor, glycoprotein Ib $\alpha$ (GPIb $\alpha$ ), under static and flow conditions [8]. The study of platelet activation induced by a protein-coated surface with QCM-D showed its ability to monitor platelet spreading [9]. vWF immobilized QCM device was used to evaluate association/dissociation processes of vWF-GPIb under shear conditions [10]. In a recent work, nanoparticle induced platelet aggregation was monitored utilizing QCM-D technology [11]. None of those studies on primary haemostasis were performed in whole blood. QCM devices have also been used to study blood coagulation[12], [13].

To the best of our knowledge, the first crucial stage of haemostasis, which is platelet adhesion to subendothelial components, such as collagen, has never been studied by means of QCM. Moreover, it is critical to use whole blood for a relevant assessment of such a process, since red blood cells play an important role [14].

This contribution introduces a new approach using a QCM biosensor that enables the study of the influence of shear rate on platelet deposition at physiologically relevant flow conditions.

\section{MATERIALS AND METHODS}

\section{A. Materials}

Phosphate Buffered Saline (PBS), Bovine Serum Albumin (BSA), N-(3-dimethylamino-propyl)-N-ethylcarbodiimide (EDC), N-hydroxysulfosuccinimide (sulfo-NHS), glutaraldehyde, ethanolamine $\mathrm{HCl} 1 \mathrm{M}$, 11-mercapto-1undecanol (11-MUOH) and 16-mercaptohexadecanoic acid (16-MHA), were purchased from Sigma Aldrich, France. Collagen Horm ${ }^{\circledR}$ (type I) and isotonic glucose solution pH 2.72.9 (SKF solution) were obtained from Takeda Austria GmbH. Mouse anti Human CD42b antibody clone AK2 was obtained from Bio-Rad Laboratories.

The samples of whole blood from two healthy male donors were provided by the Etablissement Français du Sang (EFS) in Besancon, France, in compliance with current ethical regulations in France. Whole blood was collected into $1.6 \mathrm{~mL}$ blood collecting tubes with hirudin as anticoagulant. Each donor blood was analysed for the blood cell count and tested with PFA-100 device to control the blood status on the day of the test - the former since haematocrit and platelet count influence platelet deposition; the latter to check primary haemostasis - results were within the normal ranges. Hematocrit level for donor1 was in the range $42.3 \pm 0.5 \%$ and for donor2 $41.7 \pm 0.5 \%$. Platelets count for donor1 was $280 \cdot 10^{9} / \mathrm{L} \pm 4 \%$ and for donor $250 \cdot 10^{9} / \mathrm{L} \pm 4 \%$.

Single side contacting QCMs of $14 \mathrm{~mm}$ diameter with central frequency $5 \mathrm{MHz}$ (QCM5140CrAu120-050-Q supplied by Quartz Pro AB, Jarfalla, Sweden) were used in all experiments. They were used with the fluidic cell only once for performing a single perfusion test (they are cheap and normally disposable, but, in principle, could even be recycled).

\section{B. Collagen based biointerface}

Once the vessel is injured, sub-endothelial collagen is exposed to blood and acts as a major component involved in the platelet response [15]. According to the recommendations of International Society on Thrombosis and Haemostasis (ISTH), fibrillar collagens (particularly type I and III) are the most preferable biointerfaces for primary haemostasis study [16].

The QCM gold surface was functionalized with a mixture of 11-MUOH (90\%) and 16-MHA (10\%) solution at $1 \mathrm{mM}$ concentration overnight. Afterwards, the sensor was incubated for 45 minutes with a mixture of $400 \mathrm{mM}$ EDC and $100 \mathrm{mM}$ sulfo-NHS followed by immobilization of collagen by its primary amines at room temperature overnight. In all experiments, collagen Horm ${ }^{\circledR}$ was used at a concentration of $50 \mu \mathrm{g} . \mathrm{mL}^{-1}$ in SKF solution. The biointerface was then blocked with $0.1 \%$ of BSA in sodium acetate buffer solution $10 \mathrm{mM} \mathrm{pH}$ 5.5 to prevent non-specific binding. For the deactivation of remaining free ester reactive groups, an additional treatment step with ethanolamine $\mathrm{HCl} 1 \mathrm{M} \mathrm{pH} 8.5$ was performed.

The sensor biointerface was characterized at the surface of three QCMs prepared on three different days. Each QCM surface was imaged with AFM in three different zones $(100 \times 100 \mu \mathrm{m})$ within the QCM sensing area. Representative AFM images of collagen biointerface are shown in Fig. 1. 
The thickness of collagen fibres at the sensor surface was found to be in a range $86 \pm 1 \mathrm{~nm}$, comparable to literature results [17], [18] with average surface coverage of the biosensor of 23 $\pm 2.6 \%$. The low standard deviation of coverage demonstrates a high reproducibility of the collagen immobilization process on QCM.

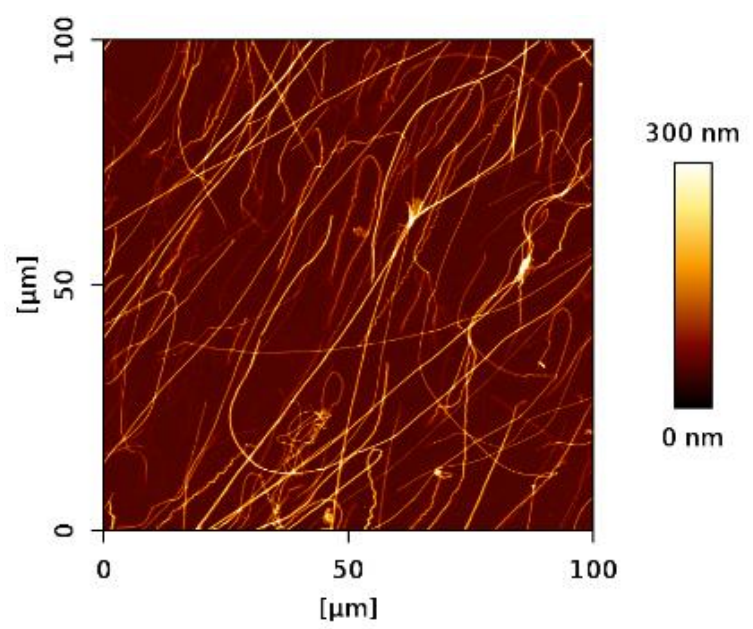

(a)
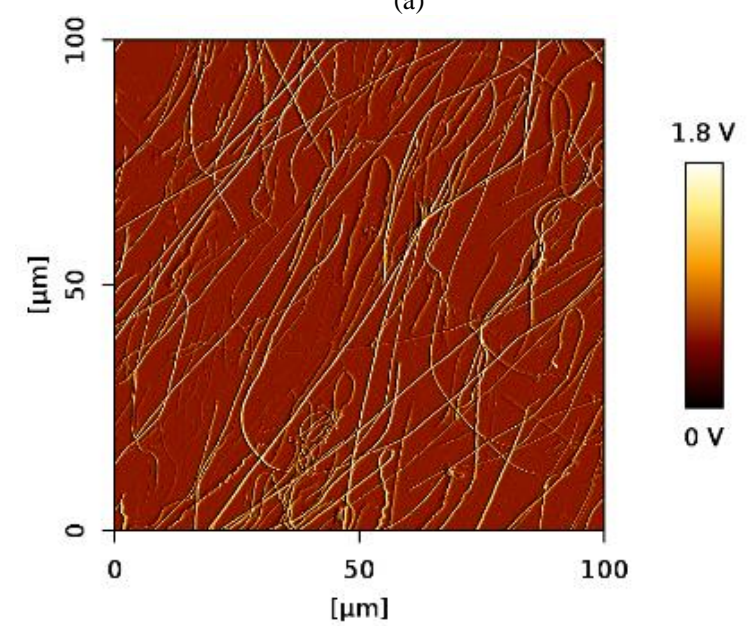

(b)

Fig. 1. AFM image of immobilized fibrillar collagen type I on QCM surface, height trace (a) and deflection trace (b).

\section{Microfluidic cell design}

The microfluidic cell was designed to obtain wall shear rates in a range of $500-1500 \mathrm{~s}^{-1}$. Fig. 2 (a) shows the manufactured and assembled biosensor. It consists of two adjacent parts made of poly(methyl methacrylate) (PMMA) with machined surface for obtaining the perfusion chamber and the inlet and outlet ports. The PMMA parts are screwed together to hold the QCM at the bottom of the chamber with a polydimethylsiloxane (PDMS) sealing ring. Both, plastics PMMA and PDMS, are not known to affect blood clotting and have an acceptable (low) cost. A printed circuit board is mounted at the bottom of the cell and provides direct connection of the QCM sensor to the impedance analyser.

A theoretical model was numerically computed with the COMSOL Multiphysics 5.3a software using the fluidic module for simulation of laminar flow. The fluidic cell height was parametrically swept and shear rate values were obtained depending on the applied inlet flow Fig. 2 (b). The height for predefined lateral geometry of the chamber regulates the flow rate at which the targeting shear rate can be reached. As it is shown in Fig. 2 (b), larger chamber height requires higher flow rate to reach the same wall shear rate that leads to overconsumption of the donor blood. On the other hand, low chamber height enables the significant reduction of blood consumption but requires strict fabrication tolerances and precise control of the flow rate to ensure the targeting shear rate conditions. We choose to use a fluidic cell height of $50 \mu \mathrm{m}$ as suggested from the literature [19] and as a value that is in agreement with existing fabrication and experimental facilities. This specific point is in accordance with the recommendations of the ISTH, validating the designed perfusion chamber for the assessment of primary haemostasis [20].

\section{Experimental conditions}

Perfusion tests were completed at a predefined flow rate for each test. They were performed at room temperature, which was kept stable in the range $23-24^{\circ} \mathrm{C}$. Perfusion time for all experiments was set to five minutes, a duration commensurate with standard bleeding test time. Blood perfusion tests were completed within two hours after blood collection. Those conditions follow the current recommendations for blood perfusion tests [19], [20]. Of note, however, the circular shape of the QCM sensor governs the in-plane geometry of the fluidic chamber, deviating from the recommended rectangular parallel plate flow chamber. Platelet deposition kinetics was studied with anticoagulated whole blood of two healthy donors. For the same donor, the tests were performed on three separate days to assess the reproducibility of the results. The tests for the range of shear rates were performed on the same day with the same donor's blood and with three QCM sensors prepared on the same day to ensure equal conditions for all biointerfaces.

In order to avoid uneven distribution of blood cells in the chamber, the microfluidic circuit was built by minimizing tubing length to $20 \mathrm{~cm}$ and by avoiding dead volumes. The injection of whole blood and PBS were performed separately with two lines with controlled syringe pumps, which were set to the same continuous flow rate. A microfluidic valve was used to switch the inlet of the fluidic cell to the running buffer or to the blood perfusion line under bubble free conditions. The blood sample was loaded into a sterile syringe with the amount required for the experiment $(0.46-1.37 \mathrm{~mL})$. Experiments were performed with a continuous flow, physiologically relevant for small vessels where pulsing is negligible. Actually, continuous flow mode is a commonly accepted condition to study the platelets adhesion in primary haemostasis [21].

\section{E. Experimental setup}

A schematic representation of the experimental setup is shown in Fig. 2 (c). 
The QCM admittance magnitude and phase were measured with Keysight 4990A impedance analyser. In-house developed software was used to get a continuous readout of the impedance analyser data and do the tracking of the frequency of maximum of admittance along with admittance gain/phase recording. Real-time data recording was performed with a time interval of four seconds between measurements.

\section{F. QCM surface characterization}

Nanowizard III AFM (JPK Instruments, Germany) was used to characterize the QCM surface at nanoscale. This was

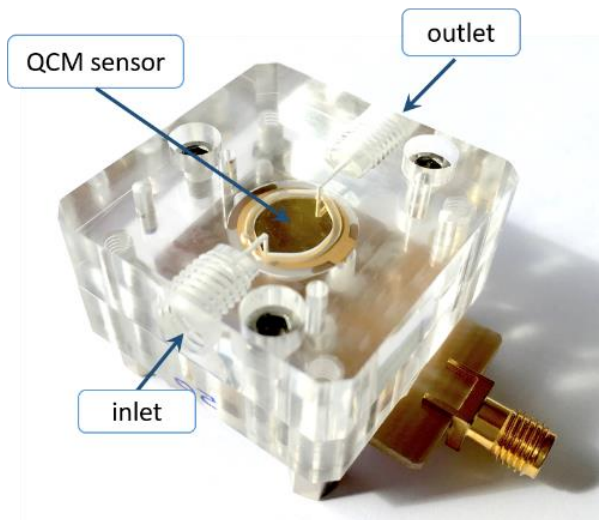

(a)

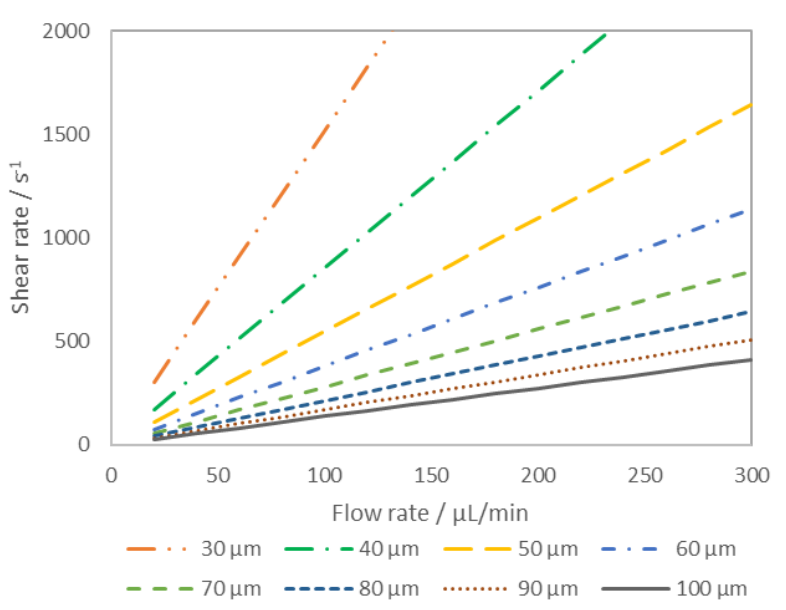

(b)

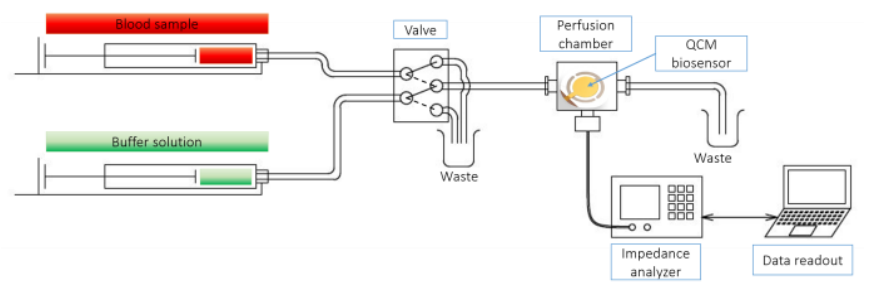

(c)

Fig. 2. Microfluidic perfusion chamber with assembled disposable QCM based biosensor: photograph (a); computation results of shear rate as a function of inlet flow for different fluidic cell heights (b); Schematic representation of the experimental setup consisting of: two separate push syringes (one loaded with blood and another with PBS buffer); microfluidic valve to switch between loading and perfusion; perfusion chamber with assembled disposable QCM biosensor; and impedance analyser with data readout by external computer (c). performed at the end of blood perfusion and after washing with PBS for five minutes and fixation with glutaraldehyde $(0.5 \%$ concentration). Several images of the QCM sensing area were taken for the maximal scanning area $100 \times 100 \mu \mathrm{m}$ using Nano World NPS-10C cantilevers made from silicon nitride with a stiffness of $0.08 \mathrm{~N} / \mathrm{m}$. AFM images were collected in a contact mode in air, at a frequency of 0.5 line/s with a resolution of 512 by 512 pixels. Height trace and deflection trace data were recorded.

Height trace scans were used to evaluate surface deposits using two metrics, average thickness and surface coverage. In this work, the average thickness is defined as the arithmetic mean value of the height trace across the $100 \times 100 \mu \mathrm{m}$ scanned area, and can be considered to represent the average volume of deposits on the sensing area. Coverage is defined as a ratio of surface covered by deposits to free surface when 0 corresponds to completely free surface and 1 to full surface coverage. Deflection signal measurements were only used for visualization purposes showing a better contrast inside the deposits.

Additionally, optical microscope images were obtained with a ZEISS AXIO IMAGER VARIO microscope using $\times 10$ objective and image stitching for recording the full QCM surface.

\section{RESULTS}

\section{A. Fluidic cell}

We first checked the distribution of deposits on the QCM surface. Deposit tests were performed for two shear rates corresponding to the extreme values of the studied range $\left(500 \mathrm{~s}^{-1}\right.$ and $\left.1500 \mathrm{~s}^{-1}\right)$. Microscope images were compared with the distribution of the shear rate obtained numerically, as shown in Fig. 3.

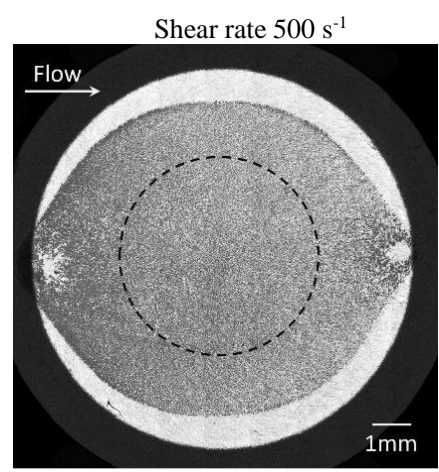

(a)

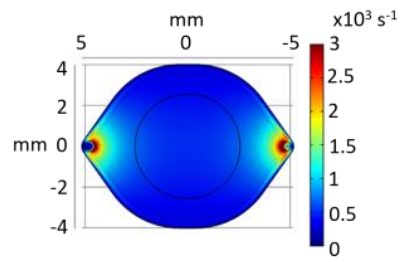

(c)

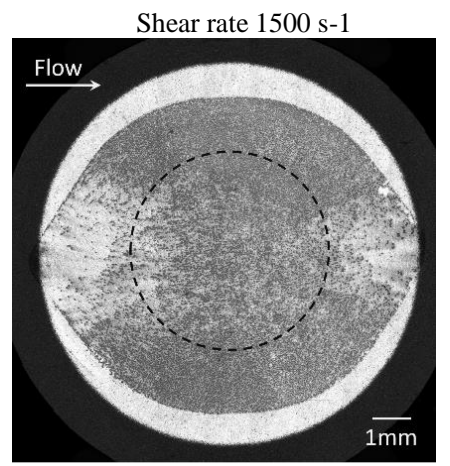

(b)

(d)

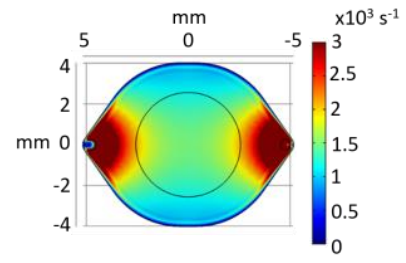

Fig. 3. Optical microscope images of the QCM biointerface with deposited platelets at two shear rates $500 \mathrm{~s}^{-1}$ (a) and $1500 \mathrm{~s}^{-1}$ (b). Black dashed circle shows the sensing area. Results of numerical computation of shear rate distribution for the experimental fluidic cell for the same conditions corresponding to shear rates $500 \mathrm{~s}^{-1}$ (c) and $1500 \mathrm{~s}^{-1}$ (d) with a circle highlighting the same sensing area. 


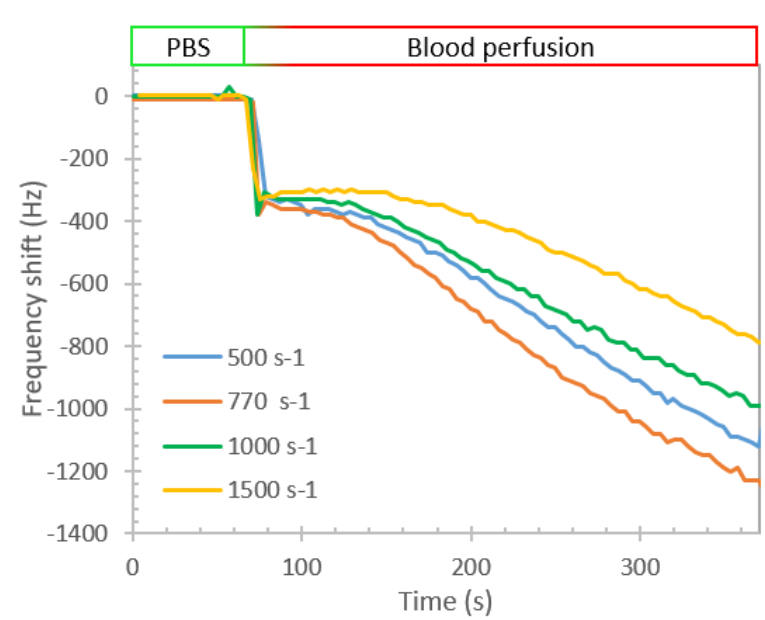

(a)

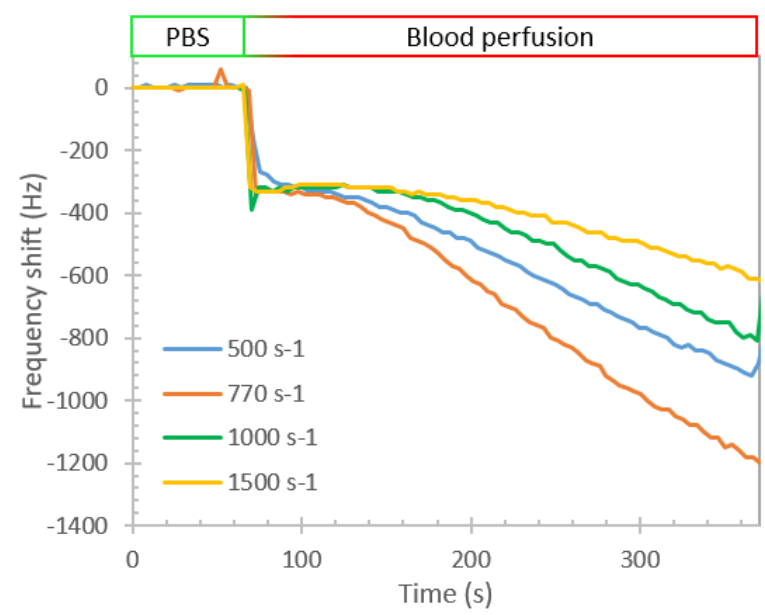

(b)

Fig. 4. QCM sensor admittance frequency shift $(\mathrm{Hz})$ recorded during the blood perfusion during five minutes for two donors (donor1 (a) and donor2 (b)) at a shear rate of $500 \mathrm{~s}^{-1}$ (blue), $770 \mathrm{~s}^{-1}$ (orange), $1000 \mathrm{~s}^{-1}$ (green) and $1500 \mathrm{~s}^{-1}$ (yellow)

The optical microscope images (Fig. 3 (a) and (b)) show that despite the existence of large irregularities of platelet deposits at the inlet and outlet of the fluidic cell, the deposits in the sensing area at the centre of the QCM was rather uniform. The numerical simulation shows the distribution of the shear rate in the chamber varies by less than $20 \%$ within the sensing area (Fig. 3 (c) and (d)) but does not significantly affects the distribution of platelet deposits (Fig. 3 (a) and (b)).

\section{B. Control experiments}

Whole blood is a complex medium that contains cells and plasma with proteins that can non-specifically interact with the biosensor interface.

First of all, non-specific interactions were prevented with passivation of the sensor interface with $0.1 \%$ BSA solution, which is a well-established means for that purpose.

Second, we assessed non-specific bindings with QCM sensors processed with the above-described protocol for the set-up of the biointerface but omitting collagen immobilization. The control data were obtained as a frequency shift during five minutes of whole blood perfusion over the control interface at

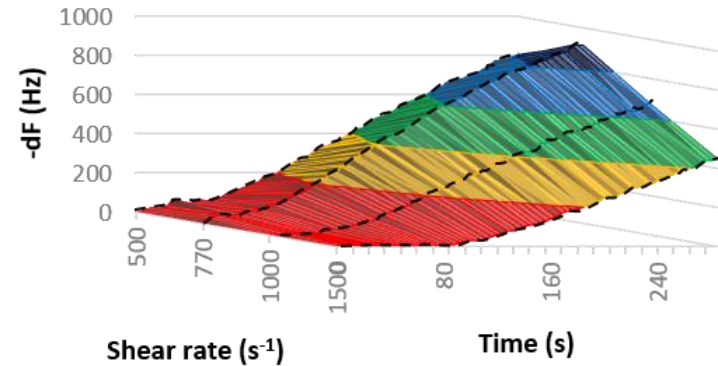

(a)

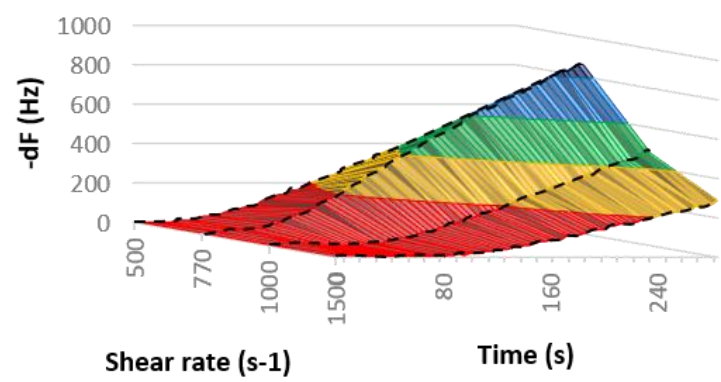

(b)

Fig. 5. 3D integrative plots obtained for donor1 (a) and donor2 (b). X-axis is the perfusion time, $\mathrm{Y}$-axis is the shear rate and $\mathrm{Z}$-axis is the absolute value of frequency shift of maximum of admittance. (The vertical colour scale has a step of $200 \mathrm{~Hz}$ that is arbitrarily chosen to be approximately $20 \%$ of the maximum frequency shift.)

the chosen shear rates. The total frequency shift corresponding to non-specific binding was below $10 \mathrm{~Hz}$ for all shear conditions.

Furthermore, we looked at non-platelet interactions with the collagen biointerface. At shear rates around $1500 \mathrm{~s}^{-1}$, vWF binding to platelet $\mathrm{GpIb}$ receptors is a prerequisite for platelet adhesion. Blocking of the receptor with a specific antibody inhibits vWF-mediated platelet adhesion. Accordingly, whole blood was incubated with a CD42b antibody directed at GpIb $(10 \mu \mathrm{g} / \mathrm{mL})$ for five minutes and then perfused during five minutes over the collagen biointerface. The total frequency shift measured after blood perfusion was $70 \mathrm{~Hz}$ on average. That value could correspond to the loading caused by any residual non-platelet interaction (see just above), specific binding of vWF protein to collagen and platelet deposits occurring despite GpIb blockade. We consider that this value defines the threshold of frequency shift where further frequency evolution is predominantly caused by platelet deposits.

\section{Real-time monitoring with QCM based biosensor}

The kinetics of platelet deposition was studied by real-time monitoring of QCM biosensor response during blood perfusion. Continuously recorded frequency shift of the QCM maximum of admittance was used as the biosensor readout parameter to monitor interactions at the biointerface. It is an integrated measurement that averages the viscoelastic loading across the QCM biointerface caused by deposited platelets and blood material.

Fig. 4 shows frequency shift of QCM biosensor recorded during the perfusion tests at four shear rates for the two donors. The sensor frequency when the biointerface is loaded with PBS 


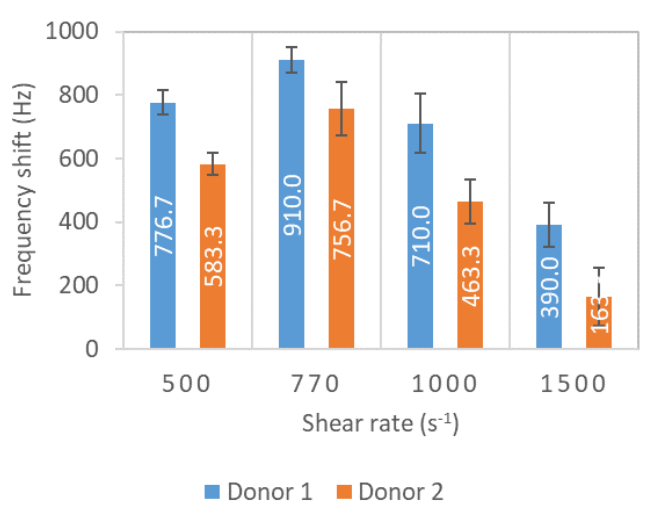

(a)

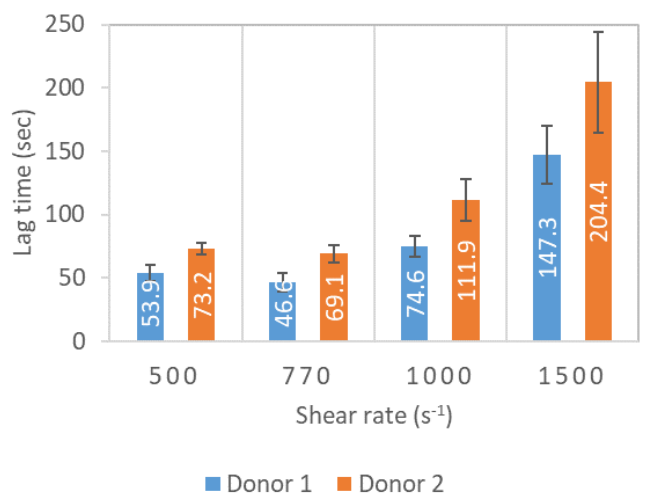

(b)

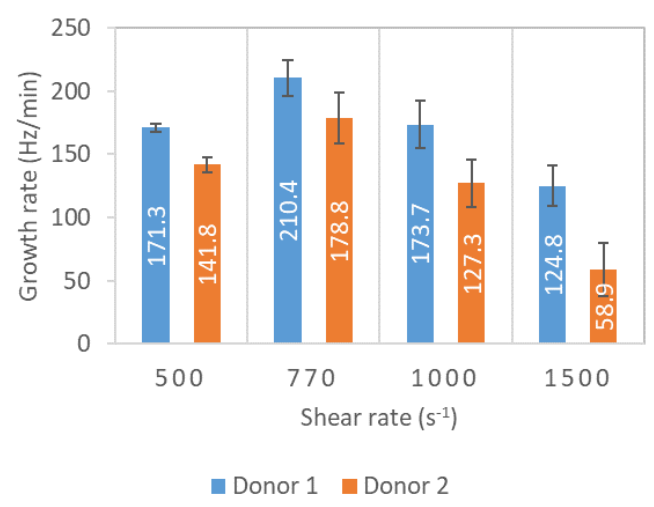

(c)

Fig. 6. Bar charts of the three defined metrics as a function of shear rates with the blood of two healthy donors: TFS (a); lag time (b); growth rate (c). Error bars show the absolute deviation from the mean value at the same shear rate. The tests were made for the same donor on three different days.

is taken as a reference to evaluate the frequency shift. When the whole blood reached the fluidic cell (at about $80 \mathrm{~s}$ as shown in Fig. 4), we observed a rapid frequency downshifting that corresponded to the change of viscosity and density between PBS and blood. Subsequent frequency shift during the blood perfusion were due to interactions at the sensor biointerface.

3D integrative representations of the same responses are shown in Fig. 5 (a)(b). These plots visualise platelet deposits over time at several shear rates in a single 3D diagram. In the integrative plots of the result of the blood perfusion, (Fig. 5), zero level corresponds to the state of the sensor when the whole blood arrived onto the collagen biointerface. An absolute frequency shift (Z-axis) likely monitors platelet deposits and their growth for the given shear rates (Y-axis) during blood perfusion (X-axis).

We choose to characterize the frequency shift over time using three simple metrics: the total frequency shift (TFS), the lag time and the growth rate. The TFS is the frequency shift between the beginning and the end of blood perfusion (difference between frequency shift at $\mathrm{t}=80 \mathrm{~s}$ and $\mathrm{t}=380 \mathrm{~s}$ ). This value corresponds to the effect of the total amount of surface deposit obtained at the end of the blood perfusion.

The lag time was defined as the time required to obtain a frequency shift of $70 \mathrm{~Hz}$ after blood injection. This threshold value corresponds to the measured value of nonspecific blood deposits in control experiments. A similar metric was previously arbitrarily introduced in flow assays with imaging as the time required to reach $1 \%$ of the surface coverage [22], [23].

The growth rate estimates the slope of the linear zone in the frequency response. It is used as an estimate of the platelet plug growth speed.

The data obtained for both donors are shown in Fig. 6. We see a steady growth of platelet deposits at each studied shear rate, with kinetics that were rather similar for both donors. The lag time was found minimal for shear rate $770 \mathrm{~s}^{-1}$, which means that for this shear rate the interactions at the surface were initiated faster at the beginning of blood perfusion. The lag time is the longest for the highest shear rate $\left(1500 \mathrm{~s}^{-1}\right)$. The TFS and the platelets plug growth rate exhibit also an evident maximum at the shear rate of $770 \mathrm{~s}^{-1}$.

\section{AFM characterization of QCM sensor surface after blood perfusion}

At the end of the perfusion tests and after washing and fixation, the QCM surfaces were characterized with AFM. Although any deposited material coming from blood could have been responsible for a frequency shift, we think it biologically plausible that the bulk of deposited blood material was made of platelets. After careful visual inspection of AFM images, we did not see any evidence for other blood cells than platelets, nor fibrin. Moreover, the dimensions of the deposited cells are fully compatible with those of platelets. Fig. 7 shows an AFM image (height trace (a) and deflection trace (b)) of the area $7 \times 7 \mu \mathrm{m}$ with a single platelet attached to collagen fibres. Measured height profile across the platelet is shown in Fig. 7(c). The single platelet spreading was $4 \mu \mathrm{m}$ and the maximal height was $270 \mathrm{~nm}$. The deflection signal of the same AFM scan provides more insights of the platelet shape. Evaluation of collagen fibres with deflection signal (Fig. 7(d)) demonstrates 60nm range spaced bands attributed to twisted fibrillar collagen, attesting the quality of imaging parameters.

Measurements of average thickness and coverage were made at the centre of the sensing area in three separate $100 \times 100 \mu \mathrm{m}$ scans to cover an area representative of the QCM sensing zone. Representative AFM images of selected platelet deposits for 


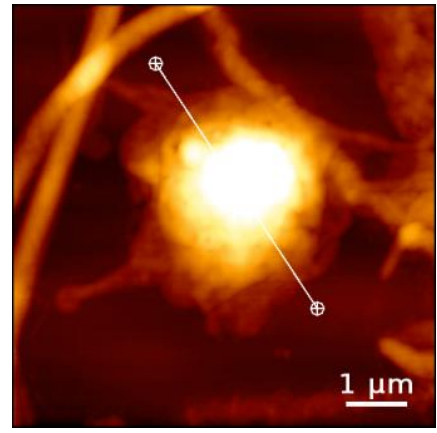

(a)

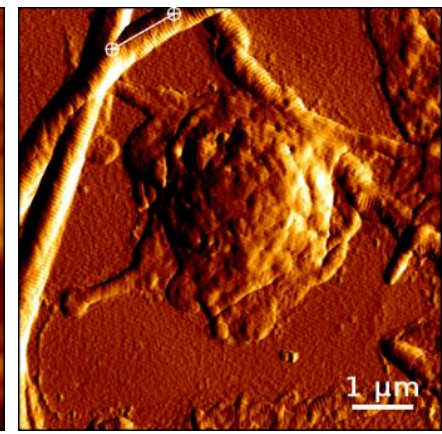

(b)

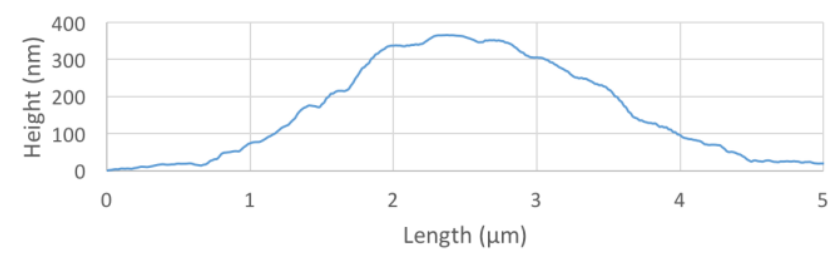

(c)

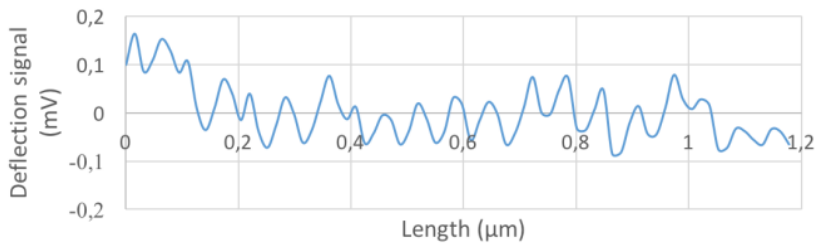

(d)

Fig. 7. AFM image height trace (a) and deflection signal (b) of the area $7 \times 7 \mu \mathrm{m}$ with a single platelet deposited at a side of collagen fibres. Measured platelet height along the white line on height trace image (c); deflection signal of collagen fibre along the highlighted line (image (b)) showing the collagen bands with $60 \mathrm{~nm}$ pitch (d).

each shear rate are shown in Fig. 8.

Results shown in Fig. 8 demonstrate a change in platelets distribution across the biointerface over the four studied shear rate cases. At 500 and $770 \mathrm{~s}^{-1}$, the number of platelet deposits was roughly the same on the collagen surface ( 8 to 10 platelet deposits on $100 \times 100 \mu \mathrm{m}^{2}$ ). However at $770 \mathrm{~s}^{-1}$, the platelet deposits were more elongated and slightly bigger, explaining the increase in coverage value. At $1000 \mathrm{~s}^{-1}$ and $1500 \mathrm{~s}^{-1}$, the number and size of platelet deposits decreased, forming more localised platelet aggregates as confirmed by coverage calculation. We still note that for all shear rates that the platelet deposits height reached $1.5 \mu \mathrm{m}$, which would correspond to several stacked platelets (up to five), a single one having a height of $280+/-30 \mathrm{~nm}$.

A summarized account of data for platelet deposits average thickness and coverage for both donors over the range of studied shear rates is shown in Fig. 9. We observe that for the two donors, both metrics show again a maximum at a shear rate of $770 \mathrm{~s}^{-1}$.

The behaviour of shear regulated platelet deposits followed the same pattern for both donors. The absolute values of the metrics at a fixed shear rate, recorded on three separate days, presented variations shown with error bars.

\section{DISCUSSION}

The described acoustic biosensor enables real-time label-free monitoring of the kinetics of initial platelet plug formation over a range of shear rates as they physiologically occur in blood
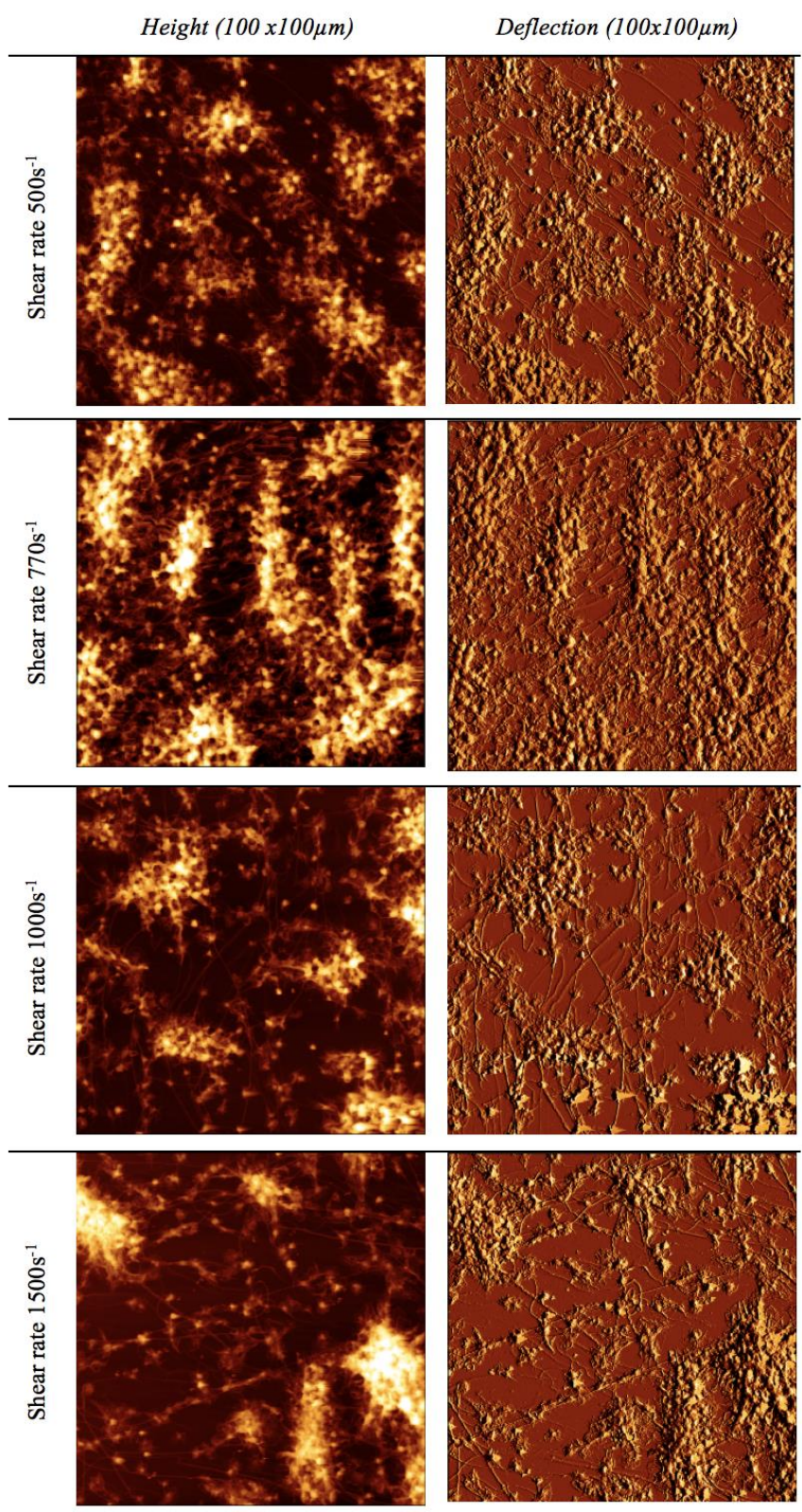

Fig. 8. Representative AFM images 100x100 $\mu \mathrm{m}$ size taken in the centre of QCM detection area after blood perfusion tests at shear rates $500 \mathrm{~s}^{-1}, 770 \mathrm{~s}^{-1}$, $1000 \mathrm{~s}^{-1}$ and $1500 \mathrm{~s}^{-1}$. Height traces (left column, maximum of $\mathrm{z}$ scale is $1.4 \mu \mathrm{m}$ ) and corresponding deflection traces (right column).

microcirculation $\left(500-1500 \mathrm{~s}^{-1}\right)$. The initial study with blood sample from healthy donor demonstrates the repeatability of the biosensor as shown in the low standard deviation of total frequency shift, lag time and growth rate during the triplicate experiments. The observed variations can be primarily attributed to the variability of the whole blood composition over separate days (for each donor, the three completed tests were conducted on three different days), which depends on many factors including the physical state of the donor, the regular uptake of meals, the blood water content, etc. The high sensitivity of the sensor is evidenced by the large difference in kinetics characteristics for small change in rheological conditions.

In our study, we proposed to use the QCM device for detailed 


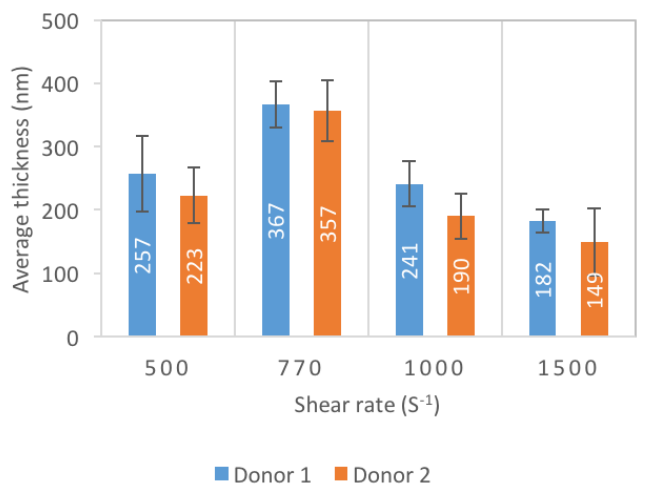

(a)

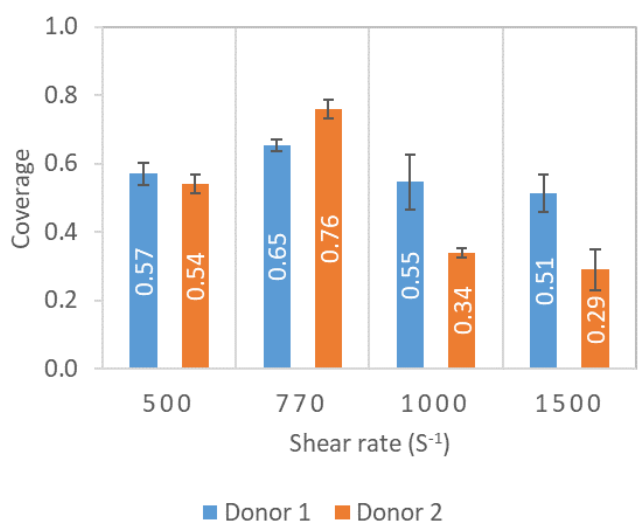

(b)

Fig. 9. Bar charts of average thickness of platelet deposits (a) and platelets surface coverage (b) obtained from AFM characterization of the QCM surface after 5 min perfusion of blood from two healthy donors at shear rates $500 \mathrm{~s}^{-1}$, $770 \mathrm{~s}^{-1}, 1000 \mathrm{~s}^{-1}, 1500 \mathrm{~s}^{-1}$. Error bars show the absolute deviation from the mean value at the same shear rate tests made for the same donor on three different days.

assessment of primary haemostasis, integrating effects of platelet deposit, platelet aggregation and initial blood plug formation. We assume that the bulk of blood material depositing on the collagen biointerface is bona fide made of platelets, both as adherent platelets and as aggregates, as suggested by the AFM images. A previous study has addressed the problem of QCM loading with viscoelastic layer at the interface with liquid [24]. They showed that the QCM fundamental mode frequency shifts quasi-linearly with the thickness of a uniform viscoelastic layer up to several micrometres. Accordingly, QCM TFS can be considered a direct measure of the total amount of platelet adhering to the sensing surface. For the AFM data, the defined average thickness is directly linked to the platelet volume and thus should be strongly correlated with TFS. To discuss this point, we have compared in Fig. 10 the AFM data (average thickness (a) and coverage (b)) with the TFS after 5 minutes blood perfusion with the QCM biosensor.

The linear correlation between the data shows that the biosensor response is in agreement with both average thickness and coverage of platelet deposits. However, the match is not perfect and the situation is more complex. In fact, both whole blood and platelets impose acoustic loading causing frequency shift. Whole blood exhibits a liquid loading of QCM that

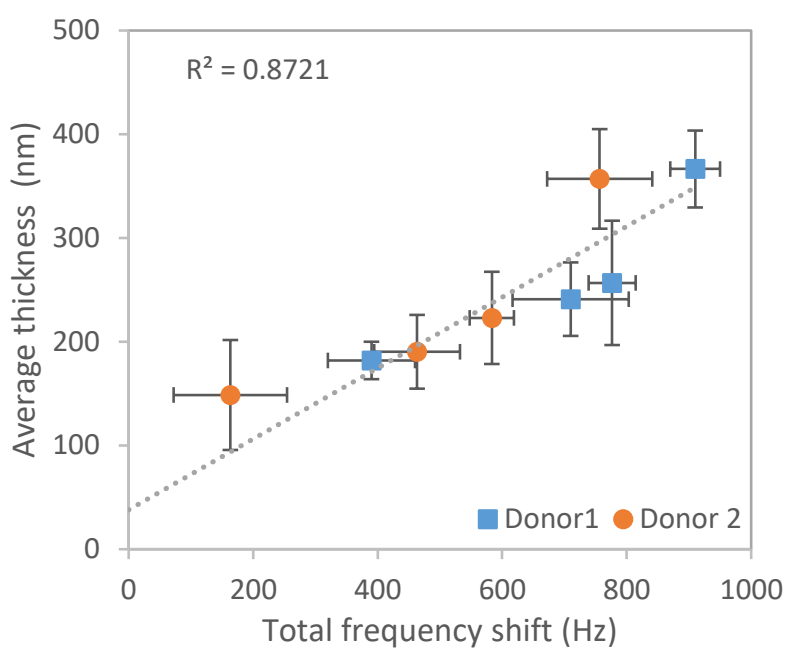

(a)

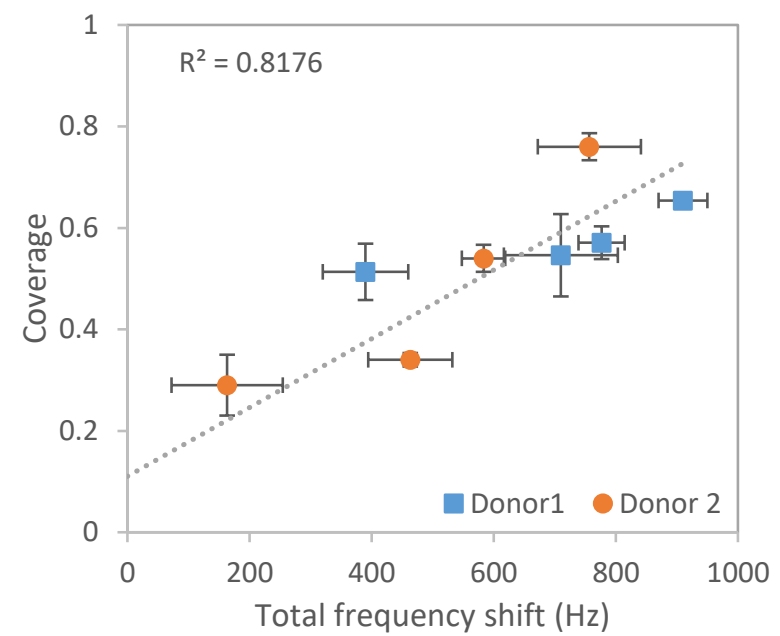

(b)

Fig. 10. Comparative analysis of recorded total frequency shift and obtained average thickness (a) and coverage (b) for both donors. Error bars show the absolute deviation from the mean value at the same shear rate tests made for the same donor on three different days (the dashed line is a linear fit of the data with coefficient of determination $\mathrm{R}^{2}$ ).

depends on viscosity and density of the liquid medium at the biointerface, which is approximated by Kanazawa and Gordon equation [25]. Frequency shift of QCM for rigidly attached solid monolayer is approximated with Sauerbrey relation [26] that is commonly described as a mass loading. Platelets on the other hand impose viscoelastic loading, which cannot be addressed as a mass because of the viscosity contribution to the total frequency shift [24], [27]-[30].

The determination coefficients in Fig. 9 revealed that the linear fit is better with the average thickness than with the coverage. In fact coverage, as commonly reported with imaging or fluorescent sensors [22], [31], is not a perfect gauge of platelet deposit as required for primary haemostasis assessment. One of the reasons lies in the fact that coverage based sensor cannot evaluate the growth of platelet layer thickness particularly after full coverage is reached. In each of our experiments the maximum height of platelet deposits reached $1.5 \mu \mathrm{m}$, which is much larger than for a single platelet. Our biosensor is able to measure the full amount of platelet deposits, 
a distinguishing feature that is important for evaluating essential mechanisms of primary haemostasis from the initial platelet adhesion to haemostatic platelet plug growth.

Moreover, our sensor enables fine recording of the kinetics of the platelet attachment to the surface (Fig. 5) bringing new opportunities for evaluating primary haemostasis. We have shown that the lag time is already a key parameter that brings information on the initial step of primary haemostasis, but the analysis of the complete kinetics could probably reveal other important characteristics of primary haemostasis.

Our results confirmed that shear rate influences platelet deposition, and evidenced a non-linear behaviour with an extremum for most metrics at a shear rate around $770 \mathrm{~s}^{-1}$. This observation underlines the complexity of the phenomena, linked to both platelet adhesion and accumulation. Under flow conditions, shear rate markedly influences platelet adhesion [22], [32], [33]. At shear rate below $500 \mathrm{~s}^{-1}$ platelets adhere to collagen by interaction with the $\alpha_{2} \beta_{1}$ platelet integrin [34] with little role if any for the vWF - GpIb interaction [35]. At shear rates in the 1000-2000 s $\mathrm{s}^{-1}$ range, it is well established that the latter interaction is a prerequisite for initial adhesion [36], [37]. The high shear rate flow forces vWF conformational changes to assist the interaction with platelet GpIb receptors [38]. Established link with vWF enables the initial tethering of platelets so that slow interactions of the platelet integrin $\alpha_{2} \beta_{1}$ with collagen can proceed, eventually resulting in firm platelet adhesion [39]-[41]. Aggregation (homotypic adhesion of platelets) is built on adherent platelets basically through platelet activation and changes in the integrin $\alpha_{I I b} \beta_{3}$ that permits the binding of its ligands, bridging adjacent platelets. The observed maximum at $770 \mathrm{~s}^{-1}$ among the four shear rates we studied could be explained as being the result of a balanced contribution of processes favouring and impending platelet adhesion and accumulation. Previous studies of shear-dependent platelet plug formation onto collagen type I showed that the surface coverage with platelets depends on shear conditions [18], [32], [33], [45]. By contrast with those previous studies, our approach is based on acoustic sensing principle that enables the determination of the amount of deposited platelet without labelling and tedious and costly imaging.

\section{CONCLUSIONS}

In this work, we proved the relevance of the acoustic biosensor approach for primary haemostasis assessment. First, the biosensor is able to use anticoagulated flowing whole blood in rheological conditions (flow-rate, chamber dimension, shearrate) relevant to haemostasis. Second, the biosensor is sensitive to the amount of deposited platelet and provides rich information on the attachment kinetics. The three introduced metrics, namely average thickness, lag time and growth rate, give a simple way to represent the complete kinetics of platelet deposit.

Improving the reliability of the biosensor will require studying the formation of the biointerface, and for example the effect of collagen fibres orientation with respect to blood flow. Its performance may also be enhanced by introducing parallel chambers of different geometries to measure in the same experiment the kinetics at different shear rates.

In fact, studying the kinetics of platelet attachment at different shear rates is expected to give a better insight into the complex phenomenon of platelet haemostatic plug formation, opening the way to a more detailed analysis of patient's primary haemostasis in laboratory medicine.

\section{REFERENCES}

[1] A. J. Gale, “Continuing Education Course \#2: Current Understanding of Hemostasis," Toxicol. Pathol., vol. 39, no. 1, pp. 273-280, Jan. 2011.

[2] M. Li, N. A. Hotaling, D. N. Ku, and C. R. Forest, "Microfluidic Thrombosis under Multiple Shear Rates and Antiplatelet Therapy Doses," PLoS One, vol. 9, no. 1, p. e82493, Jan. 2014.

[3] A. D. Michelson, "Methods for the Measurement of Platelet Function,” Am. J. Cardiol., vol. 103, no. 3, pp. 20A-26A, Feb. 2009.

[4] P. Harrison et al., "The PFA-100R: a potential rapid screening tool for the assessment of platelet dysfunction," Clin. Lab. Haematol., vol. 24, no. 4, pp. 225-232, Aug. 2002.

[5] R. Paniccia, R. Priora, A. A. Liotta, and R. Abbate, "Platelet function tests: a comparative review.," Vasc. Health Risk Manag., vol. 11, pp. 133-48, 2015.

[6] S. Sinn et al., "Platelet aggregation monitoring with a newly developed quartz crystal microbalance system as an alternative to optical platelet aggregometry," Analyst, vol. 135, no. 11, p. 2930, Oct. 2010.

[7] C. M. Larkin et al., "Platelet microaggregation in sepsis examined by quartz crystal microbalance with dissipation technology," Platelets, vol. 29, no. 3, pp. 301-304, Apr. 2018.

[8] H. Sun, P. Wang, M. Liu, and J. Xu, "A QCM-Based Lab-on-a-Chip Device for Real Time Characterization of Shear-Induced Platelets Adhesion and Aggregation," in ASME 2012 10th International Conference on Nanochannels, Microchannels, and Minichannels, 2012, p. 17.

[9] J. Fatisson, S. Mansouri, D. Yacoub, Y. Merhi, and M. Tabrizian, "Determination of surface-induced platelet activation by applying timedependency dissipation factor versus frequency using quartz crystal microbalance with dissipation," J. R. Soc. Interface, vol. 8, no. 60, pp. 988-997, Jul. 2011.

[10] M. J. Santos-Martínez, A. Prina-Mello, C. Medina, and M. W. Radomski, "Analysis of platelet function: Role of microfluidics and nanodevices," Analyst, vol. 136, no. 24. Royal Society of Chemistry, pp. 5120-5126, 21-Dec-2011.

[11] M. J. Santos-Martinez et al., "The use of quartz crystal microbalance with dissipation (QCM-D) for studying nanoparticleinduced platelet aggregation," Int. J. Nanomedicine, vol. 7, pp. 243$255,2012$.

[12] M. Hussain et al., "QCM-D surpassing clinical standard for the dose administration of new oral anticoagulant in the patient of coagulation disorders," Biosens. Bioelectron., vol. 104, pp. 15-20, May 2018.

[13] T. P. Vikinge et al., "Comparison of surface plasmon resonance and quartz crystal microbalance in the study of whole blood and plasma coagulation," Biosens. Bioelectron., vol. 15, no. 11-12, pp. 605613, Dec. 2000.

[14] J. W. Weisel and R. I. Litvinov, "Red blood cells: the forgotten player in hemostasis and thrombosis," J. Thromb. Haemost., vol. 17, no. 2, pp. 271-282, Feb. 2019.

[15] R. W. Farndale, J. J. Sixma, M. J. Barnes, and P. G. De Groot, "The role of collagen in thrombosis and hemostasis," Journal of Thrombosis and Haemostasis, vol. 2, no. 4. John Wiley \& Sons, Ltd, pp. 561-573, 01-Apr-2004.

[16] J. W. M. HEEMSKERK, K. S. SAKARIASSEN, J. J. ZWAGINGA, L. F. BRASS, S. P. JACKSON, and R. W. FARNDALE, "Collagen surfaces to measure thrombus formation under flow: possibilities for standardization," J. Thromb. Haemost., vol. 9, no. 4, pp. 856-858, Apr. 2011.

[17] A. Stylianou, D. Yova, and E. Alexandratou, "Investigation of the influence of UV irradiation on collagen thin films by AFM imaging," Mater. Sci. Eng. C, vol. 45, pp. 455-468, Jan. 2014.

[18] T. Li et al., "Studies of chain substitution caused sub-fibril level 
differences in stiffness and ultrastructure of wildtype and oim/oim collagen fibers using multifrequency-AFM and molecular modeling," Biomaterials, vol. 107, pp. 15-22, Nov. 2016.

[19] R. Van Kruchten, J. M. E. M. Cosemans, and J. W. M. Heemskerk, "Measurement of whole blood thrombus formation using parallelplate flow chambers - a practical guide," Platelets, vol. 23, no. 3, pp. 229-242, May 2012.

[20] M. ROEST, A. REININGER, J. J. ZWAGINGA, M. R. KING, and J. W. M. HEEMSKERK, "Flow chamber-based assays to measure thrombus formation in vitro: requirements for standardization," $J$. Thromb. Haemost., vol. 9, no. 11, pp. 2322-2324, Nov. 2011.

[21] R. Van Kruchten, J. M. E. M. Cosemans, and J. W. M. Heemskerk, "Measurement of whole blood thrombus formation using parallelplate flow chambers a practical guide," Platelets, vol. 23, no. 3, pp. 229-242, May 2012.

[22] K. B. Neeves et al., "Sources of Variability in Platelet Accumulation on Type 1 Fibrillar Collagen in Microfluidic Flow Assays," PLoS One, vol. 8, no. 1, p. e54680, Jan. 2013.

[23] M. V Voinova, M. Rodahl, M. Jonson, and B. Kasemo, "Viscoelastic Acoustic Response of Layered Polymer Films at Fluid-Solid Interfaces: Continuum Mechanics Approach," Phys. Scr., vol. 59, no. 5, pp. 391-396, May 1999.

[24] M. V. Voinova, M. Jonson, and B. Kasemo, "'Missing mass' effect in biosensor's QCM applications," Biosens. Bioelectron., vol. 17, no. 10 , pp. 835-841, Oct. 2002.

[25] K. K. Kanazawa and J. G. Gordon, "Frequency of a quartz microbalance in contact with liquid," Anal. Chem., vol. 57, no. 8, pp. 1770-1771, Jul. 1985.

[26] SAUERBREY and G., "The use of quarts oscillators for weighing thin layers and for microweighing," Z. Phys., vol. 155, pp. 206-222, 1959.

[27] M. V. Voinova, "Modelling of the response of acoustic piezoelectric resonators in biosensor applications \&amp;amp;ndash; Part 1: The general theoretical analysis," J. Sensors Sens. Syst., vol. 4, no. 1, pp. 137-142, Apr. 2015.

[28] M. V Voinova, M. Rodahl, M. Jonson, and B. Kasemo, "Viscoelastic Acoustic Response of Layered Polymer Films at Fluid-Solid Interfaces: Continuum Mechanics Approach," Phys. Scr., vol. 59, no. 5, pp. 391-396, May 1999.

[29] R. Lucklum and P. Hauptmann, "Quartz crystal microbalance: mass sensitivity, viscoelasticity and acoustic amplification," Sensors Actuators, B Chem., vol. 70, no. 1-3, pp. 30-36, Nov. 2000.

[30] R. Lucklum and P. Hauptmann, "Acoustic microsensors-the challenge behind microgravimetry," Analytical and Bioanalytical Chemistry, vol. 384, no. 3. Springer, pp. 667-682, Feb-2006.

[31] U. Schött and P. I. Johansson, "II. Bringing flow into haemostasis diagnostics.," Br. J. Anaesth., vol. 111, no. 6, pp. 864-7, Dec. 2013.

[32] M. Lehmann, K. Ashworth, M. Manco-Johnson, J. Di Paola, K. B. Neeves, and C. J. Ng, "Evaluation of a microfluidic flow assay to screen for von Willebrand disease and low von Willebrand factor levels," J. Thromb. Haemost., vol. 16, no. 1, pp. 104-115, Jan. 2018.

[33] X. SHI et al., "Effects of different shear rates on the attachment and detachment of platelet thrombi," Mol. Med. Rep., vol. 13, no. 3, pp. 2447-2456, Mar. 2016.

[34] H. Ni and J. Freedman, "Platelets in hemostasis and thrombosis: Role of integrins and their ligands," Transfusion and Apheresis Science, vol. 28, no. 3. Elsevier Ltd, pp. 257-264, 01-Jun-2003.

[35] B. Savage, F. Almus-Jacobs, and Z. M. Ruggeri, "Specific synergy of multiple substrate-receptor interactions in platelet thrombus formation under flow," Cell, vol. 94, no. 5, pp. 657-666, Sep. 1998.

[36] T. A. Doggett et al., "Selectin-Like Kinetics and Biomechanics Promote Rapid Platelet Adhesion in Flow: The GPIb $\alpha$-vWF Tether Bond,” Biophys. J., vol. 83, no. 1, pp. 194-205, Jul. 2002.

[37] S. W. Schneider et al., "Shear-induced unfolding triggers adhesion of von Willebrand factor fibers.," Proc. Natl. Acad. Sci. U. S. A., vol. 104, no. 19, pp. 7899-903, May 2007.

[38] T. A. Doggett et al., "Selectin-like kinetics and biomechanics promote rapid platelet adhesion in flow: The GPlb $\alpha$-vWF tether bond," Biophys. J., vol. 83, no. 1, pp. 194-205, Jul. 2002.

[39] S. Watson, "Platelet Activation by Extracellular Matrix Proteins in Haemostasis and Thrombosis," Curr. Pharm. Des., vol. 15, no. 12, pp. 1358-1372, Apr. 2009.

[40] K. L. Sarratt, H. Chen, M. M. Zutter, S. A. Santoro, D. A. Hammer, and M. L. Kahn, "HEMOSTASIS, THROMBOSIS, AND
VASCULAR BIOLOGY GPVI and 21 play independent critical roles during platelet adhesion and aggregate formation to collagen under flow," 2005.

[41] P. E. J. van der Meijden and J. W. M. Heemskerk, "Platelet biology and functions: new concepts and clinical perspectives," Nat. Rev. Cardiol., p. 1, Nov. 2018.

[42] J. J. Sixma and P. G. de Groot, "Regulation of platelet adhesion to the vessel wall.," Ann. N. Y. Acad. Sci., vol. 714, pp. 190-9, Apr. 1994. 